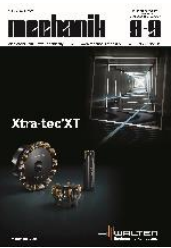

How to cite this article:

Author: Jarosław Jankowski

Title of article: „, Construction of an upper limb exoskeleton controlled by a force joystick”

Mechanik, No. 8-9 (2019)

DOI: https://doi.org/10.17814/mechanik.2019.8-9.67

\title{
Construction of an upper limb exoskeleton controlled by a force joystick
}

\section{JAROSŁAW JANKOWSKI*}

Dr inż. Jarosław Jankowski, jajan@ciop.pl, htttps://orcid.org/0000-0002-4910-7904 - Centralny instytut Ochrony Pracy-Państwowy Instytut Badawczy, Warszawa, Polska

The work describes the construction of a functional exoskeleton model of the right upper limb. Information on the key components used, i.e. drives, power supply and rack, is included. The kinematics of the mechanism are described. The developed exoskeleton is controlled by a special joystick with high rigidity made of two strain gauge sensors and allows the user to intuitively guide the effector. KEYWORDS: exoskeleton, manual control, strain gauge sensor

\section{Introduction}

Exoskeleton technology has grown rapidly in recent years. This should be explained by the development of mechanical engineering, biomedical engineering, electronics and artificial intelligence. Reviews on the structure and solutions used in exoskeletons of the upper limb are published [1-3].

At work by Rupal et al. [4], modern exoskeletons were characterized, which were compared in terms of appearance and functionality, as well as mechanical and electrical solutions. Key issues were discussed, such as actuators, control strategy, sensors, power supply methods, mechanisms and construction materials.

Also at work by Islam et al. [1], a review of achievements in the field of design and solutions used in exoskeletons of the upper limb, is presented. The drive transmission can be carried out by a rope drive, gears, pulley, harmonic gear drive, etc.

Researchers have developed different types of exoskeletons using traditional drives, e.g. electric motors or hydraulic cylinders (a detailed division was proposed by Frisoli and the team [4]). Noteworthy are works on the use of intelligent materials, e.g. electro-rheological fluids (ER, ERF), magneto-rheological fluids (MR, MRF) and shape memory alloys (SMA).

Sohn et al. [5] reviewed the applications of intelligent materials. The team focused on three materials and their properties: ERF, MRF and SMA. Actuators (smart actuators) that use intelligent materials have relatively low weight and volume compared to conventional solutions. In contrast, SMA wire actuators [6] (SMA wire actuators) can more accurately imitate the human muscle.

Materials used to make exoskeleton constructions must be relatively light. Rehman [7] proposed the whole arm ETS-MARSE made of aluminum. Copaci and the team [8] have developed an exoskeleton with an innovative design that uses intelligent materials with shape memory (SMA cables). The exoskeleton was a combination of ready-made and 3D-printed aluminum elements. To increase the user comfort, all internal parts in contact with the patient are covered with a soft, hypoallergenic textile material. The entire construction with actuators weighed less than $1 \mathrm{~kg}$. To supply the actuators with necessary energy, power supply (24 Vdc / $40 \mathrm{~A}$ ) was used. The power supply weight was $1.9 \mathrm{~kg}$.

Also Rupal [4] and his team pointed out that construction materials should have low density and high strength due to their functionality, such as special metal alloys (6061-T6 aluminum), non-metals, flexible textile materials and carbon fiber.

Noteworthy is the project called Titan Arm [9] - an exoskeleton designed to strengthen the strength of biceps - created by students from the University of Pennsylvania. The Titan Arm exoskeleton is almost entirely made of lightweight 6061-T6 aluminum alloy. Elements that are not directly exposed to loads have been printed in 3D technology. The construction weighs approximately $9 \mathrm{~kg}$ and is manually controlled by a joystick.

There are various trends in the techniques of communication between the user and the exoskeleton, i.e. commissioning and control. They can be divided into:

•intuitive (EMG, FMG, EEG), 
- manual (e.g. a joystick, the structure of which will be described in this work and is a continuation of the work described in paper [10]).

\section{Design and construction of the upper limb exoskeleton}

Construction of the exoskeleton intended for testing control methods began with the preparation and completion of basic elements such as: frame, drive section, control and power section as well as structural elements.

\section{Frame}

According to the assumptions described in paper [10], the exoskeleton should ensure mobility of use. The frame is responsible for this property. Selected model has a plastic part (frame), due to which low weight and a load capacity of up to $10 \mathrm{~kg}$ have been obtained. The frame is attached to the user's torso with braces with a chest welt and a waist belt. The company markings are: High Performance Adjustable Pad Set with 1606-AC Packframe.

\section{Drive selection}

Drives responsible for arm support (model H54-200-S500-R) and forearms (model H54-100-S500-R) selected by ROBOTIS (table) were selected.

TABLE. The most important parameters and properties of the drives used in the exoskeleton

\begin{tabular}{|l|c|c|}
\hline Drive model & H54-200-S500-R & H54-100-S500-R \\
\hline Power supply, V & \multicolumn{2}{|c|}{ cycloid with very little slack } \\
\hline Przekładnia & \multicolumn{2}{|c|}{ RS-485 } \\
\hline Communication interface & \multicolumn{3}{|c|}{25} \\
\hline $\begin{array}{l}\text { Continuous operation mo- } \\
\text { mentum(at rotational } \\
\text { speed 29 rpm), Nm }\end{array}$ & 44 & 732 \\
\hline Weight, g & 855 & 100 \\
\hline Power, W & 200 & \\
\hline
\end{tabular}

These engines are enclosed in a metal housing with a $54 \times 54 \mathrm{~mm}$ square section and a total length of $126 \mathrm{~mm}$ (model H54-200-S500-R) or $108 \mathrm{~mm}$ (model H54-100S500-R). The drives are operated via a shared library with a set of functions that allow programming movements using a dedicated USB Virtual COM - RS485 "U2D2" converter.

\section{Power selection}

The choice of batteries was dictated by the cell's current capacity, weight and availability. The selected Stiga battery with a mass of $760 \mathrm{~g}$ and capacity of $4 \mathrm{Ah}$ provides a power source with a voltage of max. $24 \mathrm{~V}$. A dedicated charger allows to fully charge the battery in about 1.5 hours. A double set of batteries allows for uninterrupted operation of the exoskeleton. An additional protection against damage to the batteries (low level of charge) is the voltage regulator applied due to the recommendations of the servo motor manufacturer.

\section{Management computer}

An Intel Compute Stick computer was used to operate the control system, sensors and servo motors. Selected model is based on an Intel Core m3-6Y30 dual-core/four-thread processor (clock speed 0.9-22 GHz). It is equipped with an on/off button, a power socket (USB 3.1 type C) and one USB 3.0 socket for communication with peripherals. The computer is small (only $114 \mathrm{~mm} \times 38 \mathrm{~mm} \times 12 \mathrm{~mm}$ ) and is light in weight (65 g). All peripherals are connected to the USB hub, which in turn is connected to the only USB port on the computer.

\section{Selection of structural elements and methods of their production}

To ensure the lowest possible weight and maximum durability of the exoskeleton under development, the main elements are made of carbon fiber tubes. Prototype components were made using 3D printing, FDM (fused 
deposition modeling), in a way that ensures the highest possible strength of the designed parts. Selected elements were additionally reinforced with bolted connections (mainly M3 and M4 bolts). The elements have been designed in Solidworks Professional.

\section{Kinematic scheme of the exoskeleton}

As a result of the analysis of the proposed assumptions and tests of the early phases of the created prototypes of the exoskeleton, a manipulator with a kinematic scheme is presented in fig. 1.

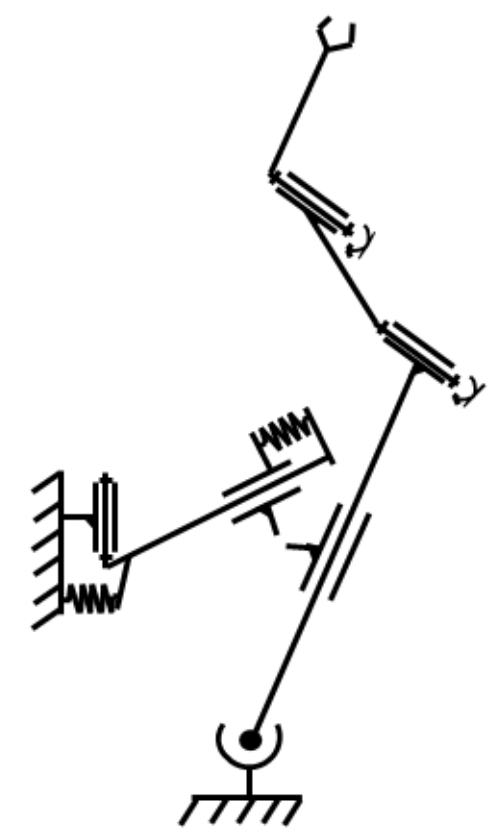

Fig. 1. Kinematic diagram of the upper limb exoskeleton

There are two attachment points in the diagram. The first, with three degrees of freedom, realized by means of a ball joint, and the second, realized with a kinematic pair of the fifth class, setting a fixed position of the axis of rotation of the part of the system responsible for the pressure of the skeleton against the user's body around the shoulder joint. The clamping system includes: a combination of two kinematic pairs of the fourth class and two springs. The end part has two drives: one for raising the arm and the other for raising the forearm. The mobility of the designed system corresponds to five degrees of freedom, i.e.: three degrees of freedom of the exoskeleton tip and two degrees of freedom of the mechanism responsible for the pressure of the "shoulder" mechanism against the human body.

\section{Exoskeleton}

Functional model of the developed exoskeleton can be divided into two segments: a part related to the frame and a movable part, i.e. a manipulator hooked in the ball joint. A manipulator hook was attached to the frame within the lap belt and a power and control section (computer) was mounted (fig. 2).

The manipulator (fig. 3) consists of two carbon fiber tubes. The first connects the manipulator with the frame, and the second acts as an arm. The forearm is made of an aluminum flat bar $50 \times 5 \mathrm{~mm}$. Cables are routed inside the pipe.

The manipulator uses straps (in two points) to keep the user's forearm in the exoskeleton. The user holds the joystick in his hand. Fig. 3 shows the connection of the rack with the supporting manipulator. 


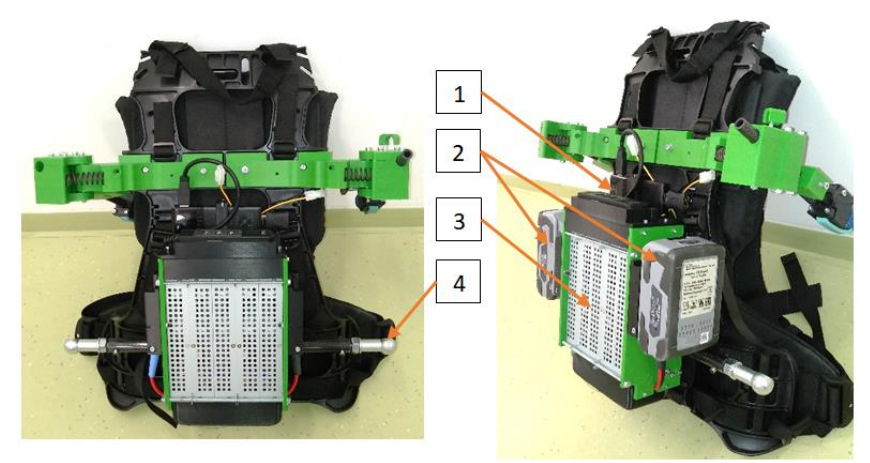

Fig. 2. Exoskeleton: a) back view, b) side view. 1 - management computer, 2 - batteries, 3 - power supply section - regulators with an openwork metal housing, 4 - rod head

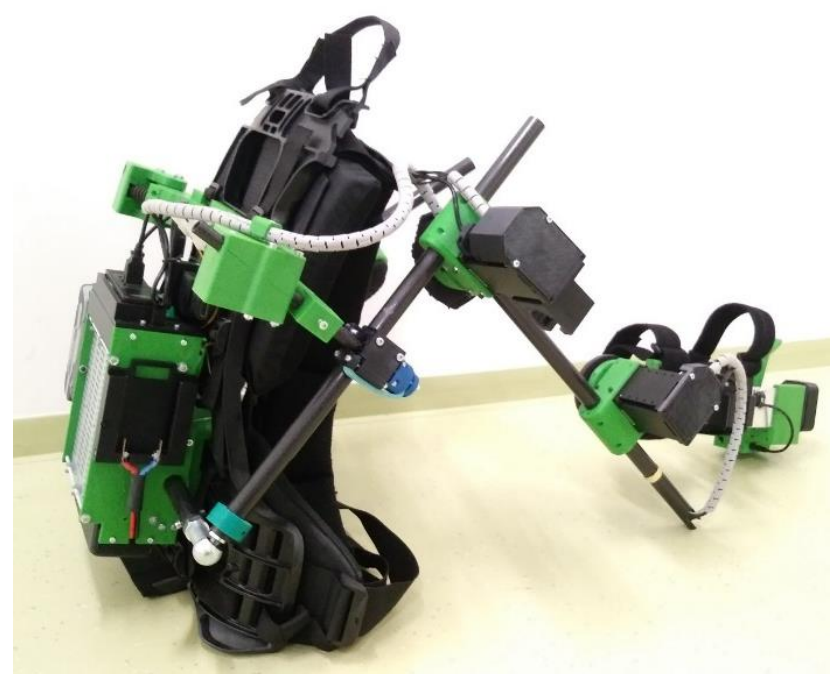

Fig. 3. Functional model of the exoskeleton

The last element connecting the user to the exoskeleton is the joystick (fig. 4). It consists of two strain gauges enclosed in a casing and a profiled handle. In the bottom of the joystick, there are dedicated signal amplifiers from strain gauges and a system with a microcontroller supporting ADC converters and a USB port.

This type of joystick - a two-axis sensor with high rigidity - allows to control the manipulator without making large movements of the hand (the user's forearm is immobilized relative to the manipulator's forearm) and is done by trying to press/pull the handle or trying to tilt the handle towards/from the user.

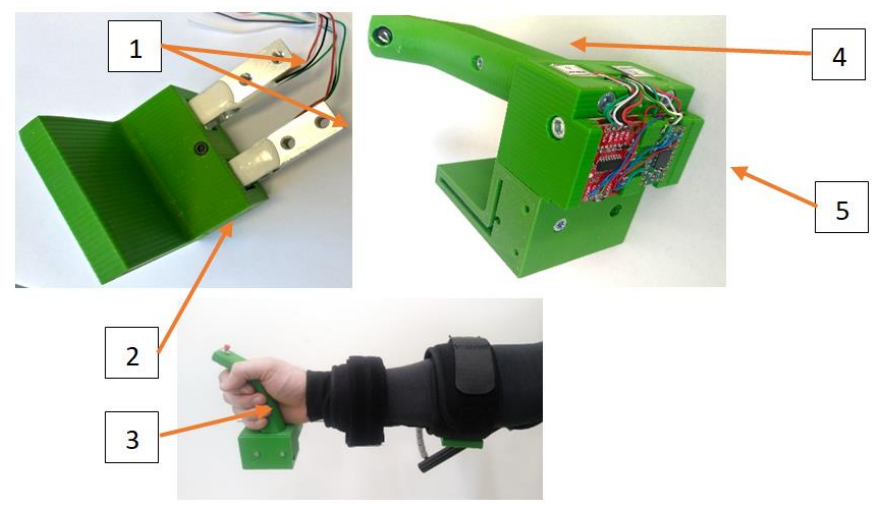

Fig. 4. Two-axis joystick. 1 - strain gauges, 2 - housing, 3 - user-operated joystick, 4 - handle, 5 - electronic circuits

Operation of the method based on manual control has been tested. The script that manages the joystick's operation after analyzing the signals ( $80 \mathrm{~Hz}$ sampling) from strain gauges concludes the direction and size of the manipulator tip position relative to the shoulder. After solving the inverse kinematics task, a new position of the manipulator effector is given, taking into account the interaction of the exoskeleton user on the joystick. 


\section{Summary}

Wearable robots can be seen as a technology that extends, supplements, replaces or increases human capabilities. The assisted limb also provides a kind of manipulator control interface. A human can supply the musclederived information to the control system, or the control system can record and process muscle effects on the exoskeleton.

The paper presents the construction of an exoskeleton that uses a high-rigidity joystick to control the trajectory of a tilted robot. Continuation of work provides for a comparison and assessment of two methods of controlling the exoskeleton under development, i.e. method based on EMG signals and method using the manual control.

The publication was prepared on the basis of results of the fourth stage of the multiannual program "Improvement of safety and working conditions", financed in 2017-2019 in the field of scientific research and development works by the National Center for Research and Development.

Program coordinator: Central Institute for Labor Protection - National Research Institute.

\section{REFERENCES}

[1] Islam M.R., Spiewak C., Rahmann M., Fareh R. "A Brief Review on Robotic Exoskeletons for Upper Extremity Rehabilitation to Find the Gap between Research Porotype and Commercial Type". Adv Robot Autom. 6 (2017): 177, DOI: $10.4172 / 2168-9695.1000177$.

[2] Ninhuijs B. van, Heide L.A. van der, Jansen J.W., Gysen B.L.J. “Overview of Actuated Arm Support Systems and Their Applications". Actuators. 2 (2013): 86-110, D0I:10.3390/act204008.

[3] Frisoli A. "Exoskeletons for upper limb rehabilitation". Rehabilitation Robotics (2018), https://www.sciencedirect.com /topics/engineering/exoskeleton-robotics, https://doi.org/10.1016/B978-0-12-8119952.00006-0.

[4] Baltej Singh Rupal et al. "Lower-limb exoskeletons: Research trends and regulatory guidelines in medical and non-medical applications". International Journal of Advanced, Robotic Systems. November-December 2017: 1-27, DOI: $10.1177 / 1729881417743554$.

[5] Jung Woo Sohn, Gi-Woo Kim, Seung-Bok Choi. A State-of-the-Art Review on Robots and Medical Devices Using Smart Fluids and Shape Memory Alloys. Appl. Sci. 8 (2018): 1928, DOI: 10.3390/app8101928.

[6] Copaci D., Serrano D., Moreno L., Blanco D. "A High-Level Control Algorithm Based on sEMG Signalling for an Elbow Joint SMA Exoskeleton". Sensors. 18 (2018): 2522, DOI:10.3390/s18082522.

[7] Zia ur Rehman M. et al.; Multiday EMG-based classification of hand motions with deep learning techniques; Sensors 2018,18, 2497; DOI: 10.3390/s18082497.

[8] Copaci D., Serrano D., Moreno L., Blanco D. "A High-Level Control Algorithm Based on sEMG Signalling for an Elbow Joint SMA Exoskeleton”. Sensors. 18 (2018): 2522, DOI:10.3390/s18082522.

[9] https://materialyinzynierskie.pl/sztuczny-egzoszkielet-wzmocni-sile-miesni/

[10] Jankowski J., Ziemek K. „Sterowanie manipulatorem nasobnym w oparciu o sygnały EMG”. Mechanik. 7 (2018): 582-584, DOI: 10.17814/mechanik.2018.7.90. 